\title{
Direct Vision Balloon Dilation for the Management of Urethral Strictures
}

\author{
Joel Gelman, M.D., ${ }^{1}$ Michael A. Liss, M.D., and Nadya M. Cinman, M.D.
}

\begin{abstract}
Urethral strictures are often initially managed with dilation using sequential metal sounds or filiform and follower dilators. While these techniques often successfully achieve at least a temporary increase to the caliber of the area of stricture, they are performed without visual guidance, and complications can include false passage and urethral perforation. We describe the first use of balloon dilator that allows the safe, controlled, and gentle and dilation of urethral strictures under direct vision.
\end{abstract}

\section{Introduction}

$\mathbf{U}$ RETHRAL DILATATION HAS BEEN USED as a management technique dating back to the 6 th century B.C. Historically, urethral dilation has been performed with rigid dilator such as Van Buren or other metal sounds or flliform and follower dilators. The objective is to increase the caliber of the narrow area of the urethra, and often dilation achieves this objective. Dilation, however, is a painful procedure that is often accompanied by bleeding, suggesting that the urethra is torn in addition to being stretched. Because the procedure is performed in a blind fashion, potential technical complications at the time of the procedure include excessive bleeding, urethral perforation with extravasation, rectal injury, and false passage.

The most common complication subsequent to the procedure is stricture recurrence, possibly with increased length because of adjacent tissue damage. ${ }^{1,2}$ In addition, we have observed that when patients are referred with bulbar strictures and have a history of sequential dilation using large sounds or followers, some were found to also have fossa navicularis strictures. It is known that fossa strictures can be caused by instrumentation using large caliber instruments in particular. ${ }^{3,4}$ This is a very unfortunate complication, because then both open bulbar repair and simultaneous fossa reconstruction are needed for definitive treatment.

Balloon dilators that advance through strictures blindly were subsequently developed, permitting the dilation of the urethra without the advancement of large caliber instruments through the fossa navicularis. ${ }^{5-7}$ It was reported that this technique was associated with a lower risk of complications and was well tolerated. ${ }^{8}$ Balloon dilators apply radial force on the stricture, with less shearing force and reduced trauma compared with the traditional rigid dilators. Less discomfort and reduced risk of spongiofibrosis are theorized as advantages of balloon dilation of the urethra. These dilators, however, are not advanced through the stricture under direct vision. Therefore, we developed the first balloon dilator that could be used to dilate urethral strictures under direct vision, and present our experience with this device.

\section{Technique}

Eleven years ago, Cook Urological produced a urethral balloon dilator according to our specifications. This balloon dilator consisted of a 5F, 80-cm open lumen, blunt tip catheter with a marked 4-cm long 10-mm diameter (approximately 30F) balloon (Cook Urologic Accent Ureteral Dilation Balloon Catheter) (Figs. 1 and 2). Subsequently, the balloon has been used as our preferred method when dilation is indicated. Our approach to patients with urethral stricture disease is to first perform urethroscopy, retrograde urethrography, and voiding cystourethrography. We then counsel our patients and explain all options and the risks and benefits of each option. Patients are advised that dilation is a reasonable option for the initial management of strictures $<1.5 \mathrm{~cm}$, and open repair generally is the best option for the management of longer or recurrent strictures if the goal is cure. Some patients and patients with multiple medical problems and radiation strictures in particular prefer to be managed with repeated dilations, however.

This technique can be used in the office using only $2 \%$ intraurethral lidocaine jelly, especially in patients who have tolerated dilation well in the past. We often perform this in the operating room under sedation, spinal, or general anesthesia, however. Patients are placed in the lithotomy position. Rigid cystoscopy is performed, because the 30F balloon does not advance through flexible cystoscopes. When the distal aspect of the stricture is visualized, a flexible tip guidewire is

\footnotetext{
${ }^{1}$ Department of Urology, University of California, Irvine, Orange, California.

${ }^{2}$ Department of Urology, North Shore Long Island Jewish Medical Center, New Hyde Park, New York.

A video demonstrating this technique is available at www.liebertonline.com/end
} 


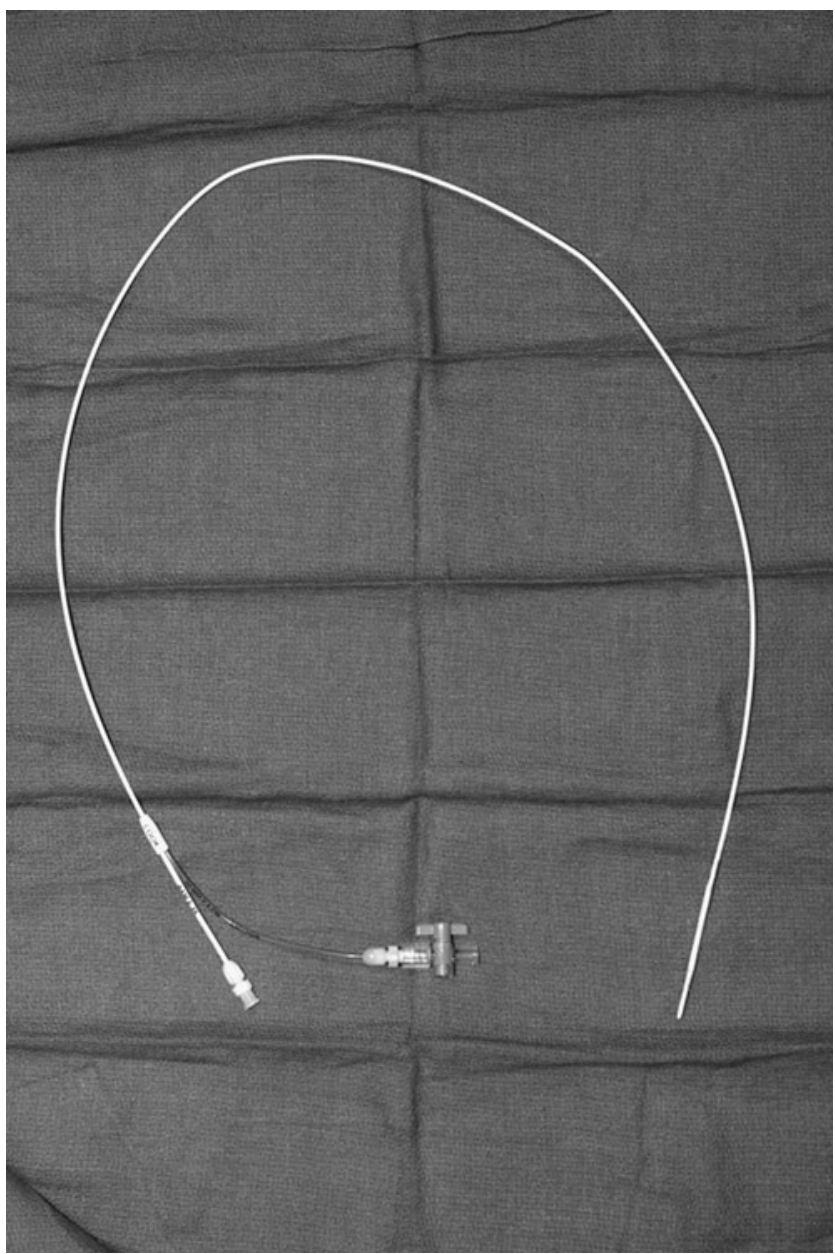

FIG. 1. Cook Balloon Dilator, which is a 5F, 80-cm open lumen, blunt tip catheter with an inflatable balloon at the distal end.

advanced. We have not encountered a situation where a guidewire would not pass easily, but would not advise proceeding if there is resistance or force needed to advance the guidewire.

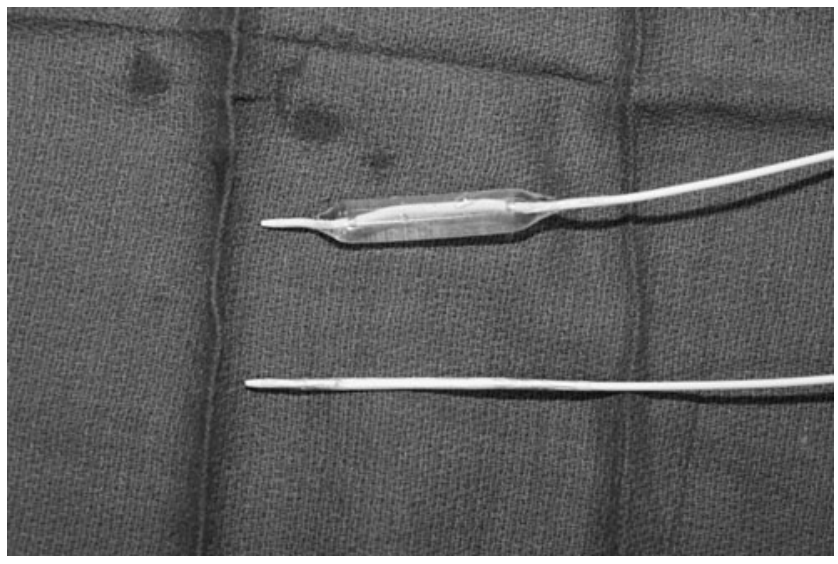

FIG. 2. Cook Balloon Dilator shown side by side in the inflated and uninflated positions.

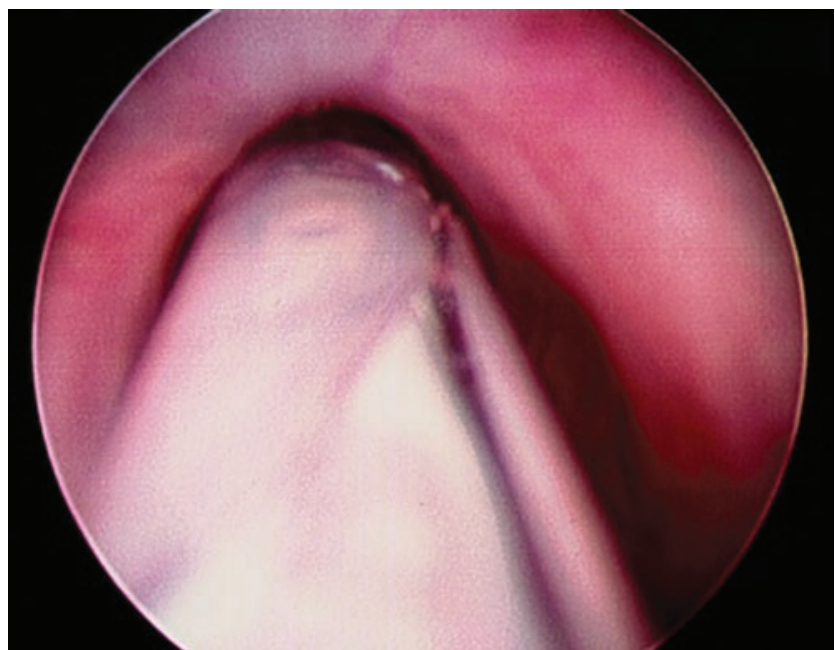

FIG. 3. Endoscopic placement of the balloon dilator across a urethral stricture.

Once access is established through the stricture, the catheter is then advanced over the guidewire under direct vision, and the balloon is positioned across the stricture (Fig. 3). The balloon is slowly inflated to 10 ATM for 5 to 10 minutes with water (maximum inflation is 12 ATM) under direct vision using a LeVeen insufflator, exerting gentle, gradual radial pressure localized to the area of stricture. Although the balloon can be inflated using radiopaque contrast to permit fluoroscopic visualization, we have not ever used fluoroscopy because we can see the urethra being adequately dilated cystoscopically (Fig. 4). The balloon is then deflated, and removed. Because the cystoscope is already adjacent to the distal aspect of the stricture, the scope can then be advanced proximally to inspect the dilated area and the urethra proximal to the stricture.

In general, dilation is not a desirable treatment for strictures longer than 1 to $2 \mathrm{~cm}$. However, if indicated, longer strictures can be dilated with the balloon dilator. In these cases, the balloon can be inflated and deflated, and then repositioned to dilate an adjacent stricture.

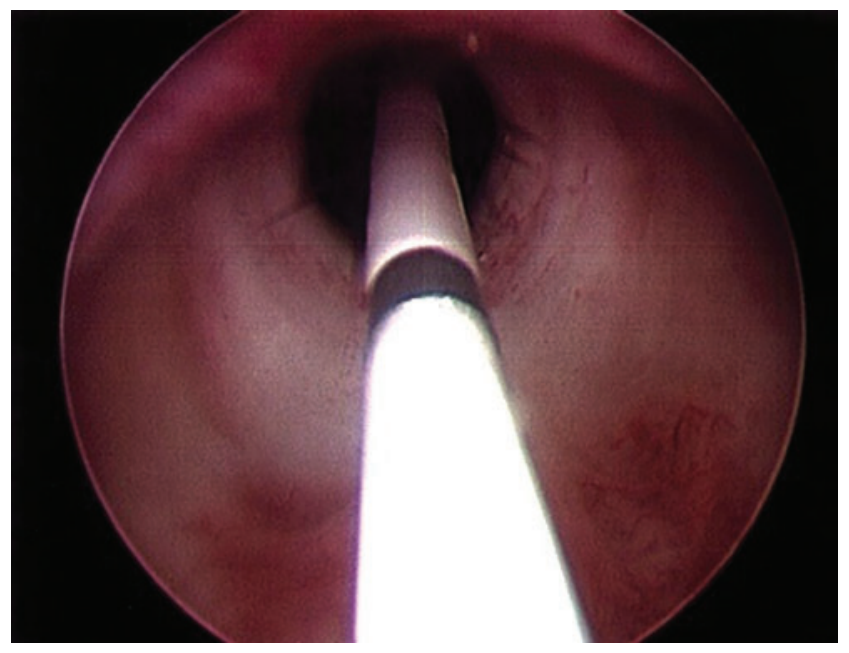

FIG. 4. Active inflation of the balloon dilator under direct vision for management of a urethral stricture. 
We have used this technique in more than 30 patients over the past 11 years without a technical procedure-related complication. This technique has been used to dilate bulbar and membranous strictures and bladder neck contractures.

\section{Equipment}

Surgical lubricant containing $2 \%$ lidocaine

Standard flexible or rigid cystoscope

Camera

Light source

Sterile water in an intravenous bag for irrigation

Cook Urologic Accent Ureteral Dilation Balloon Catheter

\section{Role in Urologic Practice}

Urethral dilation is both historically and currently a common method used to treat patients with urethral strictures. It has been clearly established, however, that dilation is often not curative. When performed to manage recurrent strictures, the cure rate approaches zero, when cure is defined as durable wide urethral patency without the need for subsequent treatment to maintain a wide caliber. ${ }^{9,10}$

It is our observation that dilation is an overused management option, because patients with urethral stricture disease are often treated with dilation when they can be cured with a properly performed urethroplasty. We are in no way suggesting that direct vision balloon dilation may offer a higher cure rate than other dilation methods. Management with periodic dilation, however, is occasionally the best choice, especially in patients who are not good candidates for urethral stricture treatment with open urethroplasty. When dilation is the best treatment plan, it is our observation that compared with blind dilation using sounds or filiforms and followers, balloon dilation under direct vision is a safe technique that is easily performed and very well tolerated.

\section{Disclosure Statement}

No competing financial interests exist.

\section{References}

1. Wong SS, Narahari R, O'Riordan A, Pickard R. Simple urethral dilatation, endoscopic urethrotomy, and urethroplasty for urethral stricture disease in adult men. Cochrane Database Syst Rev 2010;4:CD006934.

2. Devereux MH, Burfield GD. Prolonged follow-up of urethral strictures treated by intermittent dilation. Br J Urol 1970; 42:321-329.

3. Fenton AS, Morey AF, Aviles R, Garcia CR. Anterior urethral strictures: Etiology and characteristics. Urology 2005; 65:1055-1058.

4. Yee DS, Ahlering TE, Gelman J, Skarecky DW. Fossa navicularis strictures due to $22 \mathrm{~F}$ catheters used in robotic radical prostatectomy. JSLS 2007;11:321-325.

5. Russinovich NA, Lloyd LK, Griggs WP, Jander HP. Balloon dilatation of urethral strictures. Urol Radiol 1980;2:33-37.

6. Giesy JD, Finn JC, Hermann GD, et al. Coaxial balloon dilation and calibration of urethral strictures. Am J Surg 1984; 147:611-614.

7. Daughtry JD, Rodan BA, Bean WJ. Balloon dilatation of urethral strictures. Urology 1988;31:231-233.

8. MacDiarmid SA, Harrigan CT, Cottone JL, et al. Assessment of a new transurethral balloon dilation catheter in the treatment of urethral stricture disease. Urology 2000;55:408-413.

9. Steenkamp JW, Heyns CF, de Kock ML. Internal urethrotomy versus dilation as treatment for male urethral strictures: A prospective, randomized comparison. J Urol 1997;157:98-101.

10. Heyns CF, Steenkamp JW, De Kock ML, Whitaker P. Treatment of male urethral strictures: Is repeated dilation or internal urethrotomy useful? J Urol 1998;160:356-358.

Address correspondence to: Joel Gelman, M.D.

Department of Urology

University of California, Irvine

333 City Blvd. Suite 1240

Orange, CA 92868

E-mail: jgelman@uci.edu

http://www.centerforreconstructiveurology.org

DOI: 10.1089/end.2011.0307

\title{
Editorial Comment for Gelman et al.
}

\author{
Jerilyn Latini, M.D.
}

$\mathbf{T}$ HE AUTHORS PRESENT AN interesting technique of dilating urethral strictures using a commercially available balloon dilator that is positioned and inflated under direct visualization. The procedure seems straightforward and may be performed using intraurethral analgesia, sedation, spinal or general anesthesia. It carries the theoretical advantage of being more precise compared with conventional urethral dilation performed blindly.
A potential disadvantage may be that the balloon dilator is inflated for only 5 to 10 minutes, then removed. Some urologists who manage short, anterior urethral strictures with conventional balloon dilation (in which the dilator is filled with radiopaque contrast, inflated under fluoroscopy to verify that it is in the correct position, and the stricture "waist" disappears) keep the balloon inflated for 60 to 90 minutes in an effort to effectively dilate the stricture. This is in contrast to 